\title{
Evaluation of characteristics of thyroid nodules on contrast-enhanced ultrasonography: a retrospective analysis of 252 cases
}

\author{
Wen Luo $^{1 *}$, Xiao Yang ${ }^{*}$, Jiani Yuan${ }^{1}$, Lina Pang ${ }^{1}$, Peidi Zhang ${ }^{1}$, Lei Ding ${ }^{1}$, Fen Gu${ }^{1}$, Haijing \\ Liu', Yunfei Zhang ${ }^{2}$ \\ * the authors shared the first authorship
}

${ }^{1}$ Department of Ultrasound, Xijing Hospital, ${ }^{2}$ Research Institution of Bone tumor, Tangdu Hospital, Fourth Military Medical University, Xi'an, China

\begin{abstract}
Aim: This study investigated the enhancement patterns observed on contrast-enhanced ultrasound (CEUS) images for differentiating thyroid nodules. Material and methods: A retrospective review was conducted of CEUS cine loops of 252 nodules by two independent readers. Seven categories of enhancement patterns were identified: concentric hypoenhancement; heterogeneous hypoenhancement; hypoenhancement with sharp margin; homogeneous hyper/isoenhancement; hyper/isoenhancement with ring-like vascularity; island-like enhancement; and no perfusion. Associations between these patterns and the confirmed pathological/cytological outcomes (178 malignant, 74 benign) were analyzed and the sensitivity, specificity and positive predictive values (PPVs) determined. The agreement of the readers' assessments was evaluated by Kappa value. Results: For malignant nodules, the predominant 3 patterns were: concentric hypoenhancement, heterogeneous hypoenhancement and homogeneous hyper/isoenhancement. For each of these, the diagnostic specificity was above $87 \%$ and the PPV more than $85 \%$. Combining these patterns for malignancy the rates of sensitivity, specificity and PPV for reader 1 (reader 2 ) were $96.1 \%$ (98.9\%), 71.6\% (71.6\%), and 89.1\% (89.3\%), respectively. For benign nodules, the predominant 4 patterns were: hypoenhancement with sharp margin; hyper/isoenhancement with ring-like vascularity; island-like enhancement; and no perfusion. The specificity for each was above $98 \%$ and the PPV more than $70 \%$. Combining these patterns for benignity, the rates of sensitivity, specificity and PPV for reader 1 (reader 2) were $71.6 \%(71.6 \%), 96.1 \%(98.9 \%)$ and $88.3 \%(96.3 \%)$, respectively. The inter-reviewers agreement for classifying enhancement patterns was excellent $(\kappa=0.84,95 \% \mathrm{CI}: 0.79-0.89)$. Conclusions: Enhancement patterns of thyroid nodules on CEUS investigation, enable differentiation between malignant and benign lesions with good diagnostic sensitivity, specificity and PPV.
\end{abstract}

Keywords: contrast enhanced ultrasonography; thyroid nodules; benign; malignant

\section{Introduction}

The differential diagnosis of thyroid nodules is crucial for clinical management but remains challenging $[1,2]$. Ultrasonography is recommended as the first-line

Received 23.10.2019 Accepted 01.02.2020

Med Ultrason

2020, Vol. 22, No 2, 164-170

Corresponding author: Wen Luo

Department of Ultrasound, Xijing Hospital,

Fourth Military Medical University,

Xi'an, China

E-mail:lwdd1234@163.com method for diagnosis, as even small nodules (diameter $<1 \mathrm{~cm}$ ) can be easily detected [3,4]. Microcalcification, irregular margins, and a shape that is taller than wide are considered to be sensitive indicators for malignancy [5]. Most clinically significant malignancies can be identified using the international standardized IT-RADS (Thyroid Imaging, Reporting, and Data System) risk stratification system [5]. However, efforts for early stage detection have increased the number of observed nodules with atypical appearance on ultrasound. In addition, thyroiditis on ultrasound appears as an uneven echogenicity of tissue, which may interfere with the nodules' identification [6]. 
These findings observed with conventional ultrasound inevitably compromise the differentiation of thyroid nodules. Newer techniques, such as contrast-enhanced ultrasound (CEUS) have proved to improve the differential diagnosis $[7,8]$. Using intravenous injection of microbubble contrast agent, CEUS can delineate the distribution of vascularity in focal areas [9] and the differences in vascular structures between lesions and surrounding areas become clearer. CEUS has been largely used for identifying tumors in solid organs, including liver, kidney or uterus [10-13].

In a recent study Jiang et al [14] compared CEUS and conventional ultrasound and demonstrated that CEUS significantly outperformed the latter for sensitivity, specificity, positive and negative predictive values and diagnostic accordance. The values for CEUS ranged from $88 \%$ to $93 \%$, while that of conventional ultrasound were $59 \%$ to $77 \%$. This suggests that CEUS may hold promise for differentiating thyroid nodules

Enhancement patterns on CEUS have been described from different perspectives and there is no unified standard or agreement on CEUS for thyroid nodules. For example, according to the EFSUMB (European Federation of Societies for Ultrasound in Medicine and Biology) Guidelines and Recommendations, hypoenhancement is the most precise predictor of malignancy on CEUS, with high sensitivity, specificity and accuracy [13]. Liu et al [15] found that slow enhancement time and absence of rim-like enhancement were risk factors for thyroid microcarcinomas. Yuan et al [16] reported that $78.38 \%$ of malignant nodules showed inhomogeneous enhancement throughout the contrast period. Yet, the precisely differentiating benign and malignant nodules based on CEUS enhancement patterns is crucial and directly related to an accurate diagnosis.

The present study aimed to investigate the associations between enhancement patterns obtained via CEUS and histological findings of thyroid nodules.

\section{Material and methods}

\section{Patients}

The Ethics Committee of the hospital (KY20172090C-1) approved this study. All patients provided their informed consent before ultrasound investigation. Data were retrospectively analyzed for patients with thyroid nodules who underwent CEUS at our department from July 2014 to July 2016. CEUS was performed without interference (such as swallow) and the final diagnosis was confirmed by pathological results after surgery or fine-needle aspiration cytology [17]. Otherwise, nodules were excluded. For multiple nodules, the dominant nodule was evaluated.
Ten nodules were excluded due to the absence of pathological or cytological results, and another 8 nodules for unsuccessful CEUS scanning. The final study population comprised 252 nodules ( 74 benign and 178 malignant) in 252 patients ( 56 men, 196 women). The diameter of the nodules was $1.21 \pm 1.04 \mathrm{~cm}$. The final diagnosis was confirmed by pathology after surgery $(\mathrm{n}=235)$ or cytology ( $\mathrm{n}=17)$. Among the 178 malignant nodules, 2 were thyroid follicular carcinoma; one was thyroid medullary carcinoma and the remaining were papillary carcinoma $(\mathrm{n}=175)$. Of the 74 benign nodules, 45 were from nodular goiter; 10 from inflammatory changes (Hashimoto thyroiditis or granuloma) and 9 from adenoma. In addition, 10 were confirmed as benign with the evidence of follicular epithelial cells via fine-needle aspiration.

\section{Equipment and ultrasound performance}

A MyLab 90 (Esaote, Italy) ultrasound image system with an LA522 linear-array probe (4-9 MHz) was employed for CEUS examination. Patients were positioned supine with the neck exposed. A solution of the secondary generation contrast agent SonoVue (Bracco, Italy) was administered through a forearm vein in a bolus of 1.0 $\mathrm{ml}$, followed by $5 \mathrm{ml} 0.9 \%$ saline. After administration of the contrast agent, the cine loops from 0 to $90 \mathrm{~s}$ were stored. Image acquisition was performed by one sonographer with 10 years' experience in CEUS examination.

\section{Image analysis}

CEUS cine loops were independently reviewed off line by two radiologists with 10 years' experience in CEUS. The reviewers were blinded to the clinical history, images on conventional ultrasound and final diagnosis.

Enhancements on CEUS images were identified as one of the following 7 patterns: concentric hypoenhancement; heterogeneous hypoenhancement; hypoenhancement with sharp margin; homogeneous hyper/ isoenhancement; island-like enhancement; homogeneous hyper/isoenhancement with ring-like surrounding vascularity; or no perfusion (enhancement). Specifically, concentric hypoenhancement was defined as microbubbles distributed in the marginal area of the nodule and gradually expanded to the center (fig 1). Heterogeneous hypoenhancement was considered intranodular echogenicity, heterogeneous and lower than that of the surrounding thyroid tissue (fig 2). Hypoenhancement with sharp margin appeared as few microbubbles emerged within the nodule, with a clear border between the nodule and the surrounding areas (fig 3). Homogeneous hyper/isoenhancement was identified as intranodular echogenicity that was higher than or equal to that of the surrounding thyroid tissue, with a faint boundary (fig 4). Island-like enhancement showed as perfusion within the nodules within an unenhanced portion (the "lake") and an isoen- 


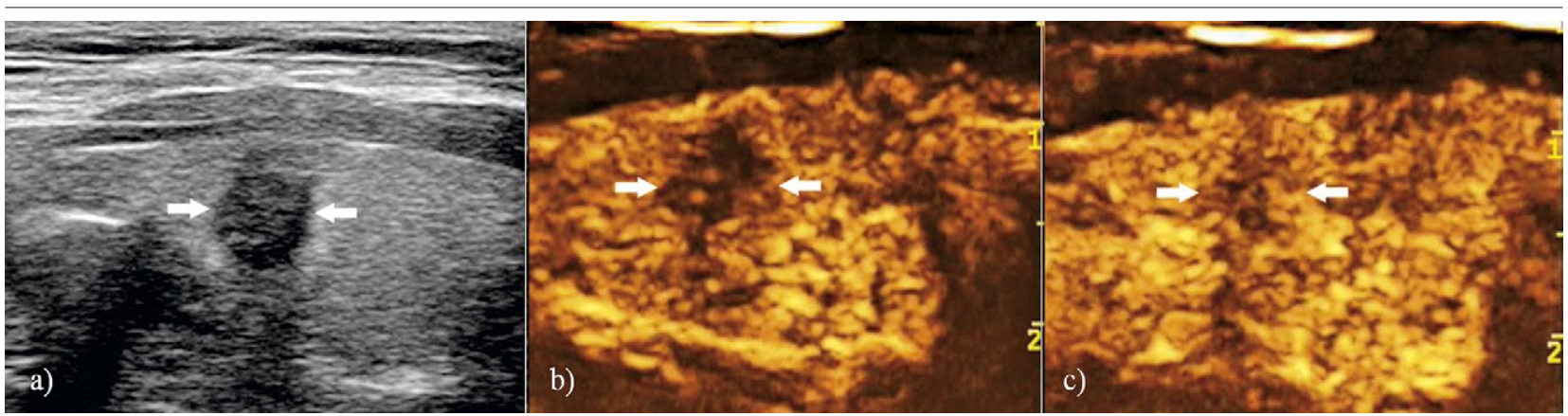

Fig 1. Woman aged 30 years with a thyroid papillary carcinoma $(0.7 \times 0.6 \mathrm{~cm})$ in the right lobe: a) conventional gray-scale ultrasound showed a hypoechoic nodule with irregular margin; b) on CEUS, the nodule appeared with hypoenhancement 15 seconds after administration of the contrast agent; c) 25 seconds after injection of microbubbles, the area of hypoenhancement within the nodule shrank (concentric hypoenhancement). Arrows indicate nodule margins.

hanced portion (the "island", fig 5) — the lake and island portions were clearly demarcated. Homogeneous hyper/ isoenhancement with ring-like surrounding vascularity showed as homogeneous enhancement detected within the nodule that was higher or equal to that of the surrounding areas, together with an enhanced ring around the nodule (fig 6). No perfusion was determined when there were no microbubbles emerging in the nodule (fig 7).

\section{Statistical analysis}

The test characteristics diagnostic sensitivity, specificity, and positive predictive value (PPV), with respective confidence intervals (CIs), were calculated for the dominant enhancement pattern that was associated with the final diagnosis (malignant or benign). Sensitivity denoted the probability of the particular pattern existing in either malignant or benign nodules. Specificity reflect-

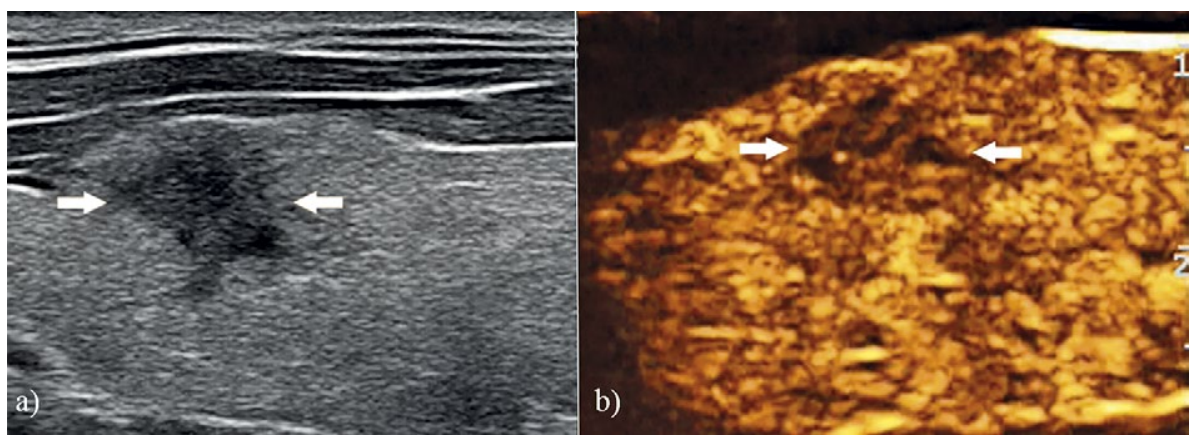

Fig 2. Man aged 55 years with thyroid granuloma $(1.0 \times 1.0 \mathrm{~cm})$ in the right lobe: a) gray-scale ultrasound showed a hypoechoic nodule with irregular margin; b) during CEUS scanning, the nodule showed heterogeneous hypoenhancement. Arrows indicate margins of the nodule.

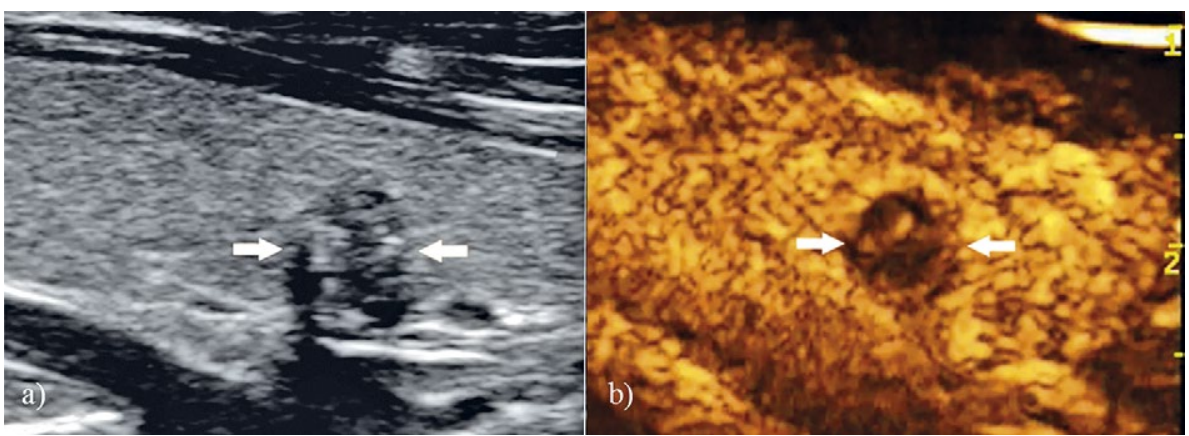

Fig 3. Man aged 53 years with nodular goiter $(0.8 \times 0.8 \mathrm{~cm})$ in the left lobe: a) gray-scale ultrasound showed hypoechoic enhancement with irregular margin; b) during CEUS scanning, the nodule showed hypoenhancement with sharp margin. Arrows indicated nodule margins. 


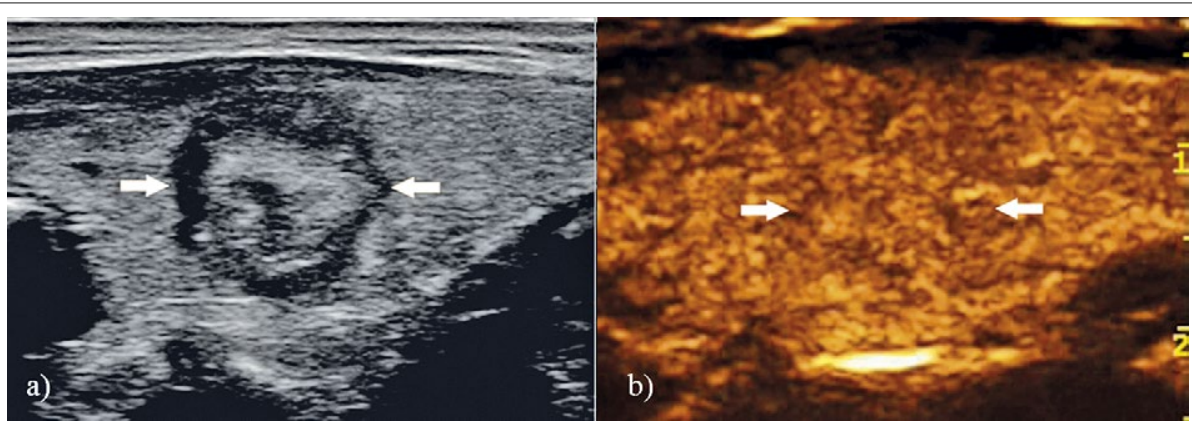

Fig 4. Woman aged 44 years with nodular goiter $(1.2 \times 1.3 \mathrm{~cm})$ in the left lobe: a) gray-scale ultrasound showed hypoechoic enhancement with irregular margin; b) during CEUS scanning, the nodule showed homogeneous isoenhancement without clear margin from the surrounding areas. Arrows indicate margins of the nodule.

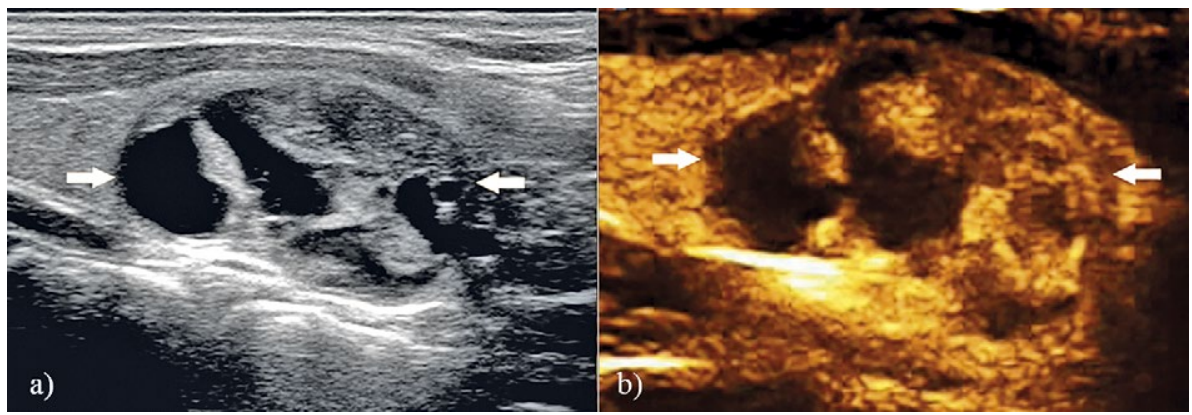

Fig 5. Woman aged 47 years with nodular goiter $(2.5 \times 1.6 \mathrm{~cm})$ in the left lobe: a) gray-scale ultrasound showed a nodule containing isoechoic and anechoic areas; b) during CEUS scanning, the nodule showed island-like enhancement. Arrows indicate margins of the nodule.

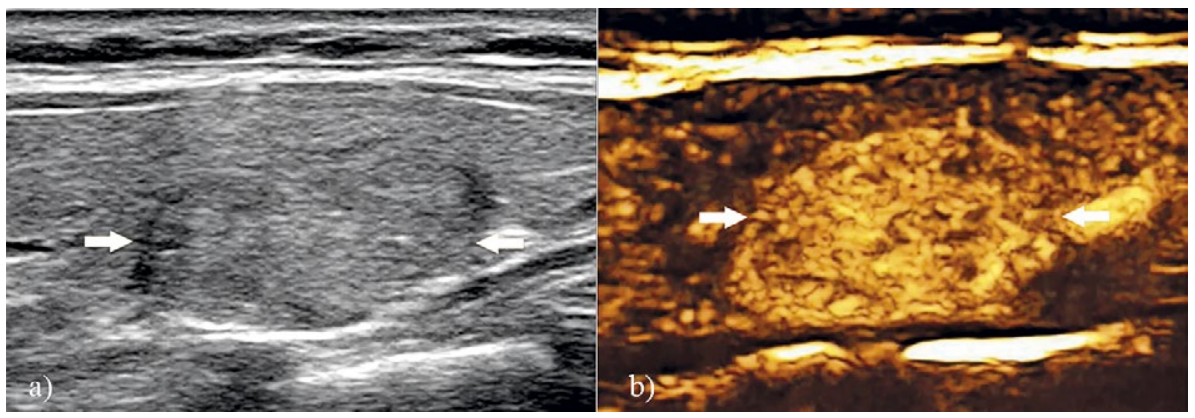

Fig 6. Woman aged 39 years with thyroid adenoma $(1.6 \times 1.2 \mathrm{~cm})$ in the left lobe: a) gray-scale ultrasound showed an isoechoic nodule with regular shape: b) during CEUS scanning, the nodule was isoenhanced with surrounding ring-like vascularity. Arrows indicate margins of the nodule.

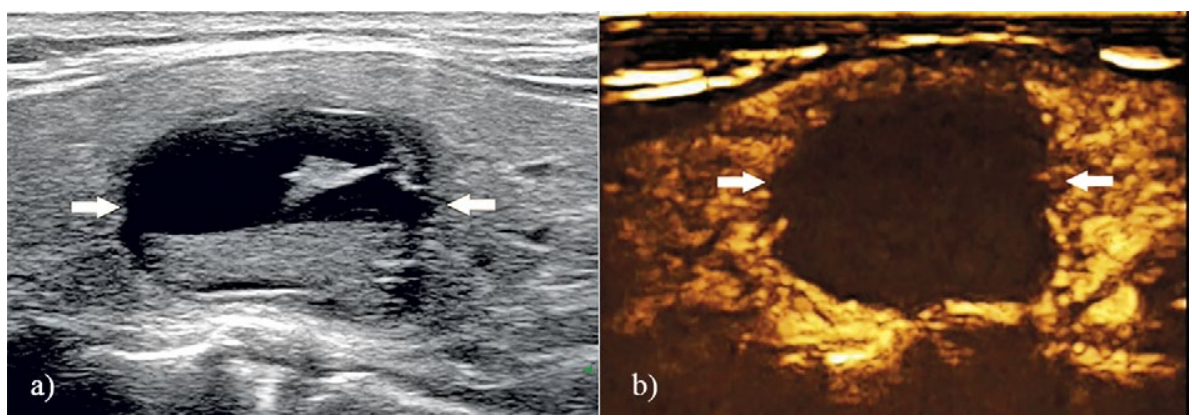

Fig 7. Woman aged 59 years with nodular goiter $(2.2 \times 1.5 \mathrm{~cm})$ in the left lobe: a) gray-scale ultrasound showed a nodule containing isoechoic and anechoic areas; b) during CEUS scanning, the nodule showed no perfusion. Arrows indicate nodule margins. 
ed the probability of the absence of a particular pattern when the nodule was either not malignant or not benign. The PPV was the probability that a nodule was diagnosed as malignant or benign if the particular pattern was observed.

SPSS 13.0 (SPSS, Chicago, IL) software was employed to perform the analyses. Inter-reviewers agreement was evaluated using kappa $(\kappa)$ values with $95 \%$ confidence interval (CI). In general, the $\kappa$ statistic was considered to represent excellent, fair-to-good and poor agreement at $\kappa>0.75,0.4-0.75$ and $<0.4$, respectively.

\section{Results}

Among the 252 nodules included in this study, 178 and 74 were confirmed malignant and benign, respectively. The nodules were classified by enhancement pattern by each reader (Table I). Assessed by reader 1 , $91.6 \%(76 / 83)$ of nodules with concentric hypoenhancement $(89.2 \%, 74 / 83$, by reader 2$), 89.4 \%(42 / 47)$ of those with heterogeneous hypoenhancement $(91.2 \%, 52 / 57$, by reader 2$)$, and $85.5 \%(53 / 62)$ of those with hyper/isoenhancement $(87.7 \%, 50 / 57$, by reader 2$)$ were confirmed as malignant. For malignant nodules, for both readers, the predominant enhancement patterns were concentric hypoenhancement, heterogeneous hypoenhancement, and hyper/isoenhancement. Assessed by reader 1, 90\% (9/10) of nodules in hyper/isoenhancement with ring-like vascularity $(92.9 \%, 13 / 14$, by reader 2$), 91.2 \%(31 / 34)$ of island-like enhancement $(100 \%, 27 / 27$, by reader 2$)$, $72.7 \%(8 / 11)$ of hypoenhancement with sharp margin $(88.9 \%, 8 / 9$, by reader 2$)$, and $100 \%(5 / 5)$ of no perfusion $(100 \%, 5 / 5$, by reader 2$)$ were confirmed as benign. For benign nodules, the most common enhancement pattern was island-like, followed by hyper/isoenhancement with ring-like vascularity, hypoenhancement with sharp margin, and no perfusion.

The sensitivity, specificity, and PPV of the dominant enhancement patterns were calculated and the results are detailed in Table II. The 3 predominate enhancement pat- terns associated with confirmed malignancy were concentric hypoenhancement, heterogeneous hypoenhancement, and hyper/isoenhancement. Combining these 3 enhancement patterns yielded diagnostic sensitivities of $96.1 \%$ and $98.9 \%$ according to readers 1 and 2, respectively; specificities of $71.6 \%$ by both readers, and PPVs of $89.1 \%$ and $89.3 \%$.

The 4 predominate enhancement patterns associated with a confirmed benign diagnosis were hypoenhancement with sharp margin, isoenhancement with ring-like vascularity, island-like enhancement, and no perfusion. If any one of these were observed in a nodule, then the nodule was predicted as benign. When this method was applied, the sensitives, specificities, and PPVs of reader 1 (reader 2) were $71.6 \%$ (71.6\%), 96.1\% (98.9\%), and $88.3 \%(96.3 \%)$, respectively.

While heterogeneous hypoenhancement was one of the predominating patterns associated with malignancy, it was also found in a few benign nodules, including granulomatous inflammation ( 3 , by both readers), nodular goiter (2, by reader $1 ; 1$ by reader 2$)$ and Hashimoto's thyroiditis $(1$, by reader 2$)$. Benign nodules with concentric hypoenhancement included Hashimoto's thyroiditis (2, by reader $1 ; 1$, by reader 2 ), granulomatous inflammation ( 2 , by both readers), and nodular goiter ( 3 , by reader 1 ; 6 by reader 2 ). Three papillary cancers were judged as island-like enhancement by reader 1 .

There were 10 nodules with a final diagnosis of inflammatory changes, i.e., 5 granulomatous inflammation and 5 Hashimoto's thyroiditis. Five enhancement patterns were associated with these nodules, including heterogeneous hypoenhancement ( 3 , reader $1 ; 4$, reader 2$)$, and concentric hypoenhancement $(4$, reader $1 ; 3$, reader 2$)$. The remaining patterns were observed in one nodule each, and the readers were in accord: hypoenhancement with sharp margin; isoenhancement; and isoenhancement with ring-like vascularity.

Overall, the inter-reviewers agreement was 0.84 (95\% CI, 0.79-0.89). Differences between the two readers were as follows: 7 nodules were evaluated as con-

Table I. Enhancement patterns of thyroid nodules (178 malignant and 74 benign) on contrast enhanced ultrasound.

\begin{tabular}{llllc}
\hline & \multicolumn{2}{c}{ Reader 1 } & \multicolumn{2}{c}{ Reader 2 } \\
\cline { 2 - 5 } & Malignant & Benign & Malignant & Benign \\
\hline Concentric hypoenhancement & 76 & 7 & 74 & 5 \\
Heterogeneous hypoenhancement & 42 & 5 & 52 & 5 \\
Hypoenhancement with sharp margin & 3 & 8 & 1 & 7 \\
Homogeneous hyper/isoenhancement & 53 & 9 & 50 & 13 \\
Hyper/isoenhancement with ring-like vascularity & 1 & 9 & 1 & 27 \\
Island-like enhancement & 3 & 31 & 0 & 5 \\
No perfusion & 0 & 5 & 0 & \\
\hline
\end{tabular}


Table II. Sensitivity, specificity, and PPV of each dominant pattern on CEUS by reader.

\begin{tabular}{|c|c|c|c|c|c|}
\hline & Enhancement pattern & Reader & Se $(\%)$ & Sp (\%) & PPV (\%) \\
\hline \multirow[t]{6}{*}{ Malignant } & \multirow[t]{2}{*}{ Concentric hypoenhancement } & 1 & 42.7 & 90.5 & 91.6 \\
\hline & & 2 & 41.6 & 89.2 & 89.2 \\
\hline & \multirow[t]{2}{*}{ Homogeneous hyper/isoenhancement } & 1 & 29.8 & 87. & 85.5 \\
\hline & & 2 & 28.1 & 90.5 & 87.7 \\
\hline & \multirow[t]{2}{*}{ Heterogeneous hypoenhancement } & 1 & 23.6 & 93.2 & 89.3 \\
\hline & & 2 & 29.2 & 93.2 & 91.2 \\
\hline \multirow[t]{8}{*}{ Benign } & \multirow[t]{2}{*}{ Isoenhancement with ring-like vascularity } & 1 & 12.2 & 99.4 & 90 \\
\hline & & 2 & 17.6 & 99.4 & 92.8 \\
\hline & \multirow[t]{2}{*}{ Island-like enhancement } & 1 & 41.9 & 98.3 & 91.2 \\
\hline & & 2 & 36.5 & 100 & 100 \\
\hline & \multirow[t]{2}{*}{ Hypoenhancement with sharp margin } & 1 & 10.8 & 98.3 & 72.7 \\
\hline & & 2 & 10.8 & 99.4 & 88.9 \\
\hline & \multirow[t]{2}{*}{ No perfusion } & 1 & 6.8 & 100 & 100 \\
\hline & & 2 & 6.8 & 100 & 100 \\
\hline
\end{tabular}

Se: sensitivity; Sp: specificity; PPV: positive predict value

centric hypoenhancement by reader 1 , but heterogeneous hypoenhancement by reader 2; 5 nodules were judged heterogeneous hypoenhancement by reader 1 , but concentric hypoenhancement by reader 2; 5 nodules were considered mixed island-like enhancement by reader 1 , but as other patterns by reader $2 ; 2$ nodules were assessed as hypoenhancement with sharp margin by reader 1 , but concentric hypoenhancement or heterogeneous hypoenhancement by reader 2; 2 nodules were depicted as isoenhancement by reader 1 but isoenhancement with ring-like vascularity by reader 2 .

\section{Discussion}

With improvements in resolution and detection, ultrasound has become an important tool for differential diagnosis of thyroid nodules [18,19], helping to avoid unnecessary surgery or biopsies. Despite the wide application of fine-needle aspiration, the diagnostic yield is limited to $80 \%$ to $99 \%$. Undetermined cytological results always result in confusion and the use of repeated fine-needle aspiration after a non-diagnostic result is still questionable [20].

We classified the CEUS patterns of the thyroid nodules in seven categories. The heterogeneous enhancement was regarded in previous studies [19-21] as the predominant pattern for malignancy but we decided to divide it into island-like enhancement (found in benign lesions) and heterogeneous hypoenhancement (found in malignant nodules), according to our observations. The most common pattern encountered in confirmed malignant nodules was the concentric hypoenhancement followed by heterogeneous hypoenhancement and homoge- neous hyper/isoenhancement patterns. In addition, about one-third of malignant nodules were depicted with hyper/ isoenhancement and the vast majority of nodules with isoenhancement were confirmed to be malignant. We found that hyper/isoenhancement in benign nodules was always associated with a vascular ring. The surrounding ring may be related to vascularity of the surface capsule or compressed surrounding tissue [8].

The island-like enhancement and hypoenhancement with sharp margin patterns mostly occurred in the nodular goiter with cystic and solid parts. We also observed that the differentiation between heterogeneous enhancement and hypoenhancement could be sometimes difficult. For this reason, we described them in detail as concentric hypoenhancement, hypoenhancement with sharp margin and heterogeneous hypoenhancement.

In the Jiang et al [12] study, hypoenhancement was used for the diagnosis of malignancy, which may indicate insufficient blood supply caused by fibrosis or calcification. However, we detected that the pattern of hypoenhancement with sharp margin always occurred in necrotic, cystic and inflammatory nodules or nodular goiter.

Due to the variable distribution of vascularity, thyroid nodules displayed diversiform patterns on CEUS, which resulted in each dominant pattern having a low diagnostic sensitivity. Therefore, combinations of enhancement patterns were analyzed. The feature of each pattern in the current study was precisely defined, so high specificity and PPV were obtained. A few nodules with an enhancement pattern that was predominantly associated with benignity were pathologically confirmed as malignant, and vice versa. For small nodules, the enhancement pattern is sometimes not clear, which may also lead to 
inter-reviewers' disagreement. Consistent with Zhang et al $[22,23]$ report, most inflammatory nodules appeared hypoenhanced, similar to that indicating malignancy, either concentric or heterogeneous. This may be due to inflammatory cells with focal fiber hyperplasia.

There were some limitations to this study. The number of medullary and follicular cancers was small and for this reason it is not possible to draw clear conclusions regarding CEUS patterns in these cases. Larger prospective studies are required.

In conclusion, we described different patterns encountered in thyroid nodules CEUS that reflect differences in the distribution of vascularity. The concentric hypoenhancement pattern typified the largest percentage of malignant nodules, but heterogeneous hypoenhancement and isoenhancement could not be ignored. Considering any of the predominant enhancement patterns associated with either malignancy or benignity yielded good diagnostic sensitivity, specificity and PPV.

\section{References}

1. Dighe M, Barr R, Bojunga J, et al. Thyroid ultrasound: state of the art. Part 2 - focal thyroid lesions. Med Ultrason 2017; 19:195-210.

2. Luo W, Zhang Y, Zhou X. Development of medical images in differentiating benign from malignant thyroid nodules. Curr Med Imaging Rev 2016;12:248-256.

3. Gamme G, Parrington T, Wiebe E, et al. The utility of thyroid ultrasonography in the management of thyroid nodules. Can J Surg 2017;60:134-139.

4. Yoo J, Ahn HS, Kim SJ, Park SH, Seo M, Chong S. Evaluation of diagnostic performance of screening thyroid ultrasonography (ultrasound) and imaging findings of screeningdetected thyroid cancer. Cancer Res Treat 2018;50:11-18.

5. Tessler FN, Middleton WD, Grant EG, et al. ACR Thyroid Imaging, Reporting and Data System (TI-RADS): White Paper of the ACR TI-RADS Committee. J Am Coll Radiol 2017; 14:587-595.

6. Grani G, Lamartina L, Ascoli V, et al. Ultrasonography scoring systems can rule out malignancy in cytologically indeterminate thyroid nodules. Endocrine 2017;57:256261.

7. Zhang JW, Chen ZJ, Gopinathan A. Focal nodular hashimoto's thyroiditis: comparison of ultrasonographic features with malignant and other benign nodules. Ann Acad Med Singapore 2016;45:357-363.

8. Tatar IG, Kurt A, Yilmaz KB, Dogan M, Hekimoglu B, Hucumenoglu S. The role of elastosonography, gray-scale and colour flow Doppler sonography in prediction of malignancy in thyroid nodules. Radiol Oncol 2014;48:348-353.

9. Zhang Y, Luo YK, Zhang MB, Li J, Li J, Tang J. Diagnostic accuracy of contrast-enhanced ultrasound enhancement patterns for thyroid nodules. Med Sci Monit 2016;22:47554764.
10. Mehta KS, Lee JJ, Taha AG, Avgerinos E, Chaer RA. Vascular applications of contrast-enhanced ultrasound imaging. J Vasc Surg 2017;66:266-274.

11. Cassinotto C, Aube C, Dohan A. Diagnosis of hepatocellular carcinoma: an update on international guidelines. Diagn Interv Imaging 2017;98:379-391.

12. Zarzour JG, Lockhart ME, West J, et al. Contrast-enhanced ultrasound classification of previously indeterminate renal lesions. J Ultrasound Med 2017;36:1819-1827.

13. Sidhu PS, Cantisani V, Dietrich CF, et al. The EFSUMB Guidelines and Recommendations for the Clinical Practice of Contrast-Enhanced Ultrasound (CEUS) in Non-Hepatic Applications: Update 2017 (Long Version). Ultraschall Med 2018;39:e2-e44.

14. Jiang J, Shang X, Wang H, Xu YB, Gao Y, Zhou Q. Diagnostic value of contrast-enhanced ultrasound in thyroid nodules with calcification. Kaohsiung J Med Sci 2015;31:138-144.

15. Liu Y, Wu H, Zhou Q, et al. Diagnostic value of conventional ultrasonography combined with contrast-enhanced ultrasonography in thyroid imaging reporting and data system (TI-RADS) 3 and 4 thyroid micronodules. Med Sci Monit 2016;22:3086-3094.

16. Yuan Z, Quan J, Yunxiao Z, Jian C, Zhu H. Contrast-enhanced ultrasound in the diagnosis of solitary thyroid nodules. J Cancer Res Ther 2015;11:41-45.

17. Haugen BR, Alexander EK, Bible KC, et al. 2015 American Thyroid Association Management Guidelines for adult patients with thyroid nodules and differentiated thyroid cancer: The American Thyroid Association Guidelines Task Force on Thyroid Nodules and Differentiated Thyroid Cancer. Thyroid 2016;26:1-133.

18. Ma HJ, Yang JC, Leng ZP, Chang Y, Kang H, Teng LH. Preoperative prediction of papillary thyroid microcarcinoma via multiparameter ultrasound. Acta Radiol 2017;58:1303-1311.

19. Baser H, Cakir B, Topaloglu O, et al. Diagnostic accuracy of thyroid imaging reporting and data system in the prediction of malignancy in nodules with atypia and follicular lesion of undetermined significance cytologies. Clin Endocrinol (Oxf) 2017;86:584-590.

20. Egset AV, Holm C, Larsen SR, et al. Risk of malignancy in fine-needle aspiration biopsy in patients with thyroid nodules. Dan Med J 2017; 64:A5320.

21. Peng QH, Niu C, Zhang M, Peng Q, Chen S. Sonographic Characteristics of Papillary Thyroid Carcinoma with Coexistent Hashimoto's Thyroiditis: Conventional Ultrasound, Acoustic Radiation Force Impulse Imaging and ContrastEnhanced Ultrasound. Ultrasound Med Biol 2019;45:471480.

22. Zhang B, Jiang YX, Liu JB, et al. Utility of contrast-enhanced ultrasound for evaluation of thyroid nodules. Thyroid 2010;20:51-57.

23. Zhang Y, Zhou P, Tian SM, Zhao YF, Li JL, Li L. Usefulness of combined use of contrast-enhanced ultrasound and TI-RADS classification for the differentiation of benign from malignant lesions of thyroid nodules. Eur Radiol 2017;27:1527-1536. 